\title{
Phonon Spectroscopy with Chirped Shear and Compressive Acoustic Pulses
}

\author{
C. L. Poyser, ${ }^{1}$ W. B. York, ${ }^{1}$ D. Srikanthreddy, ${ }^{1}$ B. A. Glavin, ${ }^{2}$ \\ T. L. Linnik, ${ }^{2}$ R. P. Campion, ${ }^{1}$ A. V. Akimov, ${ }^{1,}{ }^{*}$ and A. J. Kent ${ }^{1}$ \\ ${ }^{1}$ School of Physics and Astronomy, University of Nottingham, University Park NG7 2RD, United Kingdom \\ ${ }^{2}$ V.E. Lashkaryov Institute of Semiconductor Physics, National Academy of Sciences, Kiev 03028, Ukraine
}

(Received 16 June 2017; published 22 December 2017)

\begin{abstract}
Picosecond duration compressive and shear phonon wave packets injected into (311) GaAs slabs transform after propagation through $\sim 1 \mathrm{~mm}$ into chirped acoustic pulses with a frequency increasing in time due to phonon dispersion. By probing the temporal optical response to coherent phonons in a near surface layer of the GaAs slab, we show that phonon chirping opens a transformational route for high-sensitivity terahertz and subterahertz phonon spectroscopy. Temporal gating of the chirped phonon pulse allows the selection of a narrow band phonon spectrum with a central frequency up to $0.4 \mathrm{THz}$ for longitudinal and $0.2 \mathrm{THz}$ for transverse phonons.
\end{abstract}

DOI: 10.1103/PhysRevLett.119.255502

Terahertz (THz) and sub-THz phonon spectroscopy has become established as a powerful tool for probing and nanoscopy of various solid objects and nanostructures. The advances achieved in this field are described in a number of reviews [1-3]. An important technique for THz phonon spectroscopy is based on picosecond ultrasonics developed in the 1980s by Thomsen et al. [4] and described in detail in the recent review by Matsuda et al. [5]. Depending on the methods of phonon generation and detection, it is possible to operate with broadband $[4,5]$ or close to monochromatic phononic wave packets $[2,6,7]$. It is also possible to control phonon polarization, longitudinal or transverse, associated with compressive or shear elastic perturbations, respectively [8-13]. Information about the phonon spectrum is obtained in most of the experiments by performing a fast Fourier transform (FFT) of the optical reflectivity signal measured in the temporal domain with femtosecond resolution.

It is a challenge to increase the sensitivity of $\mathrm{THz}$ phonon spectroscopy making it a more reliable instrument for studying nano-objects. This task is beyond standard technical solutions like asynchronous optical sampling (ASOPS) [14] and requires transformational physical approaches. An appealing approach, which has not yet been realized in $\mathrm{THz}$ phonon spectroscopy, would be to exploit chirped phonon pulses. If similar improvements could be realized as in the fields of microwaves [15] and optics [16], it would significantly improve the signal-tonoise ratio and, in the long term, open prospectives for phonon frequency, phase, and polarization control. This could be used in data processing, interference, and quantum

Published by the American Physical Society under the terms of the Creative Commons Attribution 4.0 International license. Further distribution of this work must maintain attribution to the author(s) and the published article's title, journal citation, and DOI. computing, as has been proposed for chirped optical pulses [16].

In the present work, we generate sub- $\mathrm{THz}$ and $\mathrm{THz}$ upchirped shear and compressive acoustic wave packets and demonstrate the potential of the technique in phonon spectroscopy by obtaining the spectrum of the photoelastic signal from probe optical pulses. The basis of the chirp effect is phonon dispersion. Because of the curving of the acoustic dispersion, $2 \pi f=c q-\gamma q^{3}$ (in the long-wave approximation $f$ and $q$ are the phonon frequency and wave vector, $\gamma$ is the dispersion parameter, and $c$ is the sound velocity) high-frequency acoustic phonons propagate slower than low-frequency ones. As a result, the acoustic wave packet injected into a sample as a short, broadband, strain pulse spreads in time and space while propagating in the crystal and in a certain time window becomes quasimonochromatic with frequency $f$ increasing with time $t$.

For longitudinal (LA) phonons the up-chirp effect was experimentally observed in a number of crystals [17], but the chirp phenomenon has not been used for phonon spectroscopy. For transverse (TA) phonons, chirping has not previously been observed. TA phonons bringing shear perturbation to nano-objects modulate the atom displacements described by the shear modulus and thus provide, together with LA phonons, a complete characterization of the vibrational properties of a nano-object. The interaction of electrons [18,19], magnons [20-22], and photons $[11,23]$ with phonons in many types of defects and nanostructures depends on the phonon mode, with large differences for LA and TA modes due to wavelength and the different physical coupling mechanisms, e.g., deformation potential and piezoelectric electron-phonon coupling. Thus, spectroscopy using TA phonons is essential to fully characterize the interactions. By analogy with ultrasound imaging techniques, the contrast for nanoscopy 
using TA phonons in biological objects, like cells, should be higher than for LA phonons [24]. Finally, in liquids and amorphous nanolayers and structures the TA phonons should decay more rapidly than LA phonons; thus, the spectrum of their scattering or absorption could provide clearer information about the properties of disordered systems on nanometer scale.

In the case of a very short seed strain pulse, which starts to propagate in the crystal with the low-frequency sound velocity $c$, at the distance $x$ from the source corresponding to propagation time $t_{0}=x / c$, it transforms into an upchirped acoustic pulse where the displacement $u(t, x)$ is an oscillating function. For $t-t_{0} \ll t_{0}$ the frequency of the oscillations is equal to the corresponding phonon frequency and is described by [17]

$$
f(t)=\sqrt{\frac{c_{i}^{4}\left(t-t_{0}\right)}{12 \pi^{2} \gamma_{i} x}},
$$

where the index $i$ indicates phonon polarization. In the realistic case of a seed pulse with a picosecond duration, the up-chirping becomes experimentally noticeable at the minimum propagation distance $x_{\min } \approx c / \gamma q_{\max }^{3}$, where $q_{\max }$ is the maximum wave vector in the phonon wave packet.

In the experiments, we use slabs of (311) GaAs with a thickness of $0.44 \mathrm{~mm}$. The picosecond strain pulses are generated in a 30-nm-thick Al transducer deposited by electron beam evaporation on the (311) surface of the slab. The film is excited by pulses from a femtosecond laser with a tunable wavelength $(700-900 \mathrm{~nm})$ and repetition rate $80 \mathrm{MHz}$, focused to a $20-\mu \mathrm{m}$-diameter spot on the $\mathrm{Al}$ film. As well as using the $\mathrm{Al}$ transducer, experiments with direct excitation of GaAs by the frequency doubled (wavelength $\sim 400 \mathrm{~nm}$ ) optical pulses were also carried out. Because of the low-symmetry plane of GaAs, the strain pulse injected from the polycrystalline $\mathrm{Al}$ transducer into the sample possesses both compressive and shear components resulting in the propagation of quasilongitudinal (QLA), slow quasitransverse (QSTA), and fast transverse (FTA) phonons. These phonons are propagating towards the opposite surface of the slab with the phase velocities $c_{\mathrm{LA}}=5.1 \times 10^{3} \mathrm{~m} / \mathrm{s}, c_{\mathrm{QSTA}}=2.9 \times 10^{3} \mathrm{~m} / \mathrm{s}$, and $c_{\mathrm{FTA}}=$ $3.1 \times 10^{3} \mathrm{~m} / \mathrm{s}$ [19]. The wave vectors for all generated phonons are pointing exactly along [311], but elastic cubic anisotropy results in angular deviations for the propagation direction. The propagation angles from [311] towards direction [ $\overline{2} 33]$ are $11.8^{\circ}$ for QLA, $-10.7^{\circ}$ for FTA, and $-22.5^{\circ}$ for QSTA [19]. Thus, phonons with different polarizations arriving at the opposite slab surface are well separated in time and space. Acoustic pulses are detected at the opposite surface by measuring the reflectivity changes $\Delta R(t)$ of frequency doubled optical probe pulses, focused to a $20-\mu$ m-diameter spot. A photoconductive AlGaAs/ GaAs $p-i-n$ acoustic detector [25] was also used for some measurements. The experiments are carried out at low temperature $(T \approx 10 \mathrm{~K})$ in order to avoid the attenuation of $\mathrm{THz}$ phonons [26]. For details of the experimental scheme, see Supplemental Material [27].

Figure 1 demonstrates the up-chirped phonon signals for the three phonon polarizations and $t=0$ corresponds to the time when the deviation of the reflectivity starts due to the arrival of acoustic pulse. The increase of the oscillation frequency in the temporal shapes $\Delta R(t)$ [Fig. 1(a) for QSTA and FTA and Fig. 1(d) for QLA phonons] is clearly seen. The dependencies of the oscillation frequency $f$ on time are shown in the color plots of Figs. 1(b) and 1(c) for QSTA and FTA phonons, respectively. For QLA, the color maps of $f(t)$ are shown for excitation of the seed strain pulse in the Al film [Fig. 1(e)] and direct excitation of the GaAs surface with blue light [Fig. 1(f)]. The $f(t)$
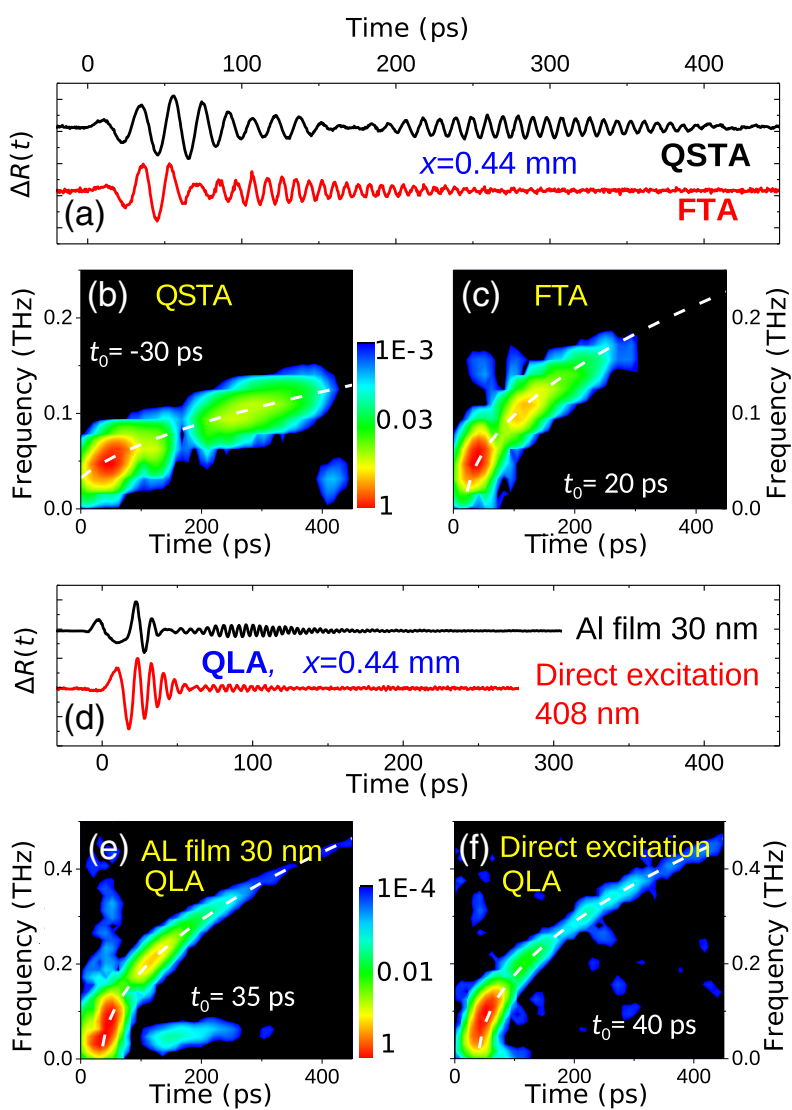

FIG. 1. (a) Temporal signals for transverse (QSTA and FTA) phonons probed with light, of wavelength $412 \mathrm{~nm}$, at the side of the GaAs slab opposite to the pump spot. (b),(c) Color maps showing the dependencies of oscillation frequency on time for QSTA and FTA phonons, respectively obtained as FFTs in a time window of 50 ps. (d) Temporal signals for QLA phonons when the seed pulse is excited in the Al film transducer (black upper curve) or directly in GaAs with blue light at $408 \mathrm{~nm}$ (red lower curve). (e),(f) The same as (b) and (c) but measured for QLA phonons with the seed pulse excited in the Al transducer or directly by blue light. The white dashed lines in (b), (c), (e), and (f) are the best fits using Eq. (1) with corresponding dispersion parameters and values for $t_{0}$. 
dependencies are obtained by performing a FFT from $\Delta R(t)$ in a 50-ps time window, centered on $t$. The white dashed lines in the color maps are the best fits of $f(t)$ using Eq. (1) with the following dispersion parameters $\gamma$ (in $\left.10^{-17} \mathrm{~m}^{2} \mathrm{~s}^{-1}\right): 2.6,3.9$, and 1.5 for QLA, QSTA, and FTA, respectively. A good agreement between the measured and calculated dependencies $f(t)$ is obtained. The numerical calculations using the strain pulses seeded from the metal film suggests that Eq. (1) is valid for all polarizations in our experimental conditions with an appropriate value of $\gamma$ [27]. The highest frequency $(\approx 0.5 \mathrm{THz})$ clearly distinguished in the chirped acoustic pulses [Fig. 1(f)] is realized for the case of direct excitation of the GaAs surface with blue light.

Figure 2(a) shows for QLA phonons, which have the highest signal amplitude (10 and 100 times higher than for QSTA and FTA, respectively), how it is possible to control the chirped pulse and corresponding $f(t)$ by changing the propagation distance $x$. We show four temporal traces measured for acoustic pulses reflected up to 6 times from the surfaces of the slab. It is seen how the acoustic pulse spreads in time while the propagation distance increases. However, the high-frequency phonons $(f>0.2 \mathrm{THz})$ are strongly damped in the multiply reflected pulse relative to the direct acoustic pulse [27].

Figure 2(b) shows how the maximum frequency in the QLA chirped pulse is increased by increasing the excitation density on the Al film. The upper curve in Fig. 2(b) corresponds to the highest density $W \sim 1 \mathrm{~mJ} \mathrm{~cm}^{-2}$ used in our experiments. At this density, nonlinear acoustic effects start to play a role $[28,29]$. The nonlinear acoustic effects are seen in Fig. 2(b) as an earlier arrival time of the QLA pulse by $10 \mathrm{ps}$ relatively to the pulses measured for lower pump power [27]. The maximum frequency which we could clearly distinguish in the oscillations of the chirped QLA pulse in our experiments for $W \sim 1 \mathrm{~mJ} \mathrm{~cm}^{-2}$ is $0.4 \mathrm{THz}$.
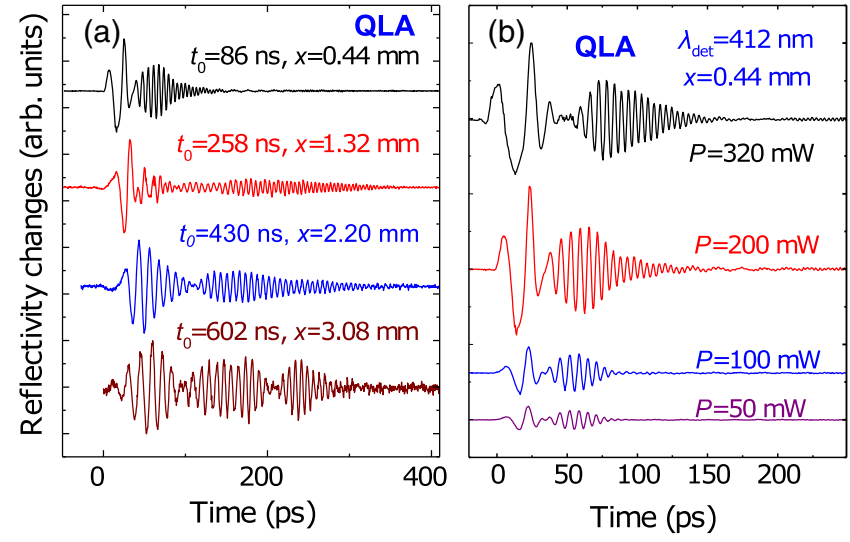

FIG. 2. (a) Chirped pulses for QLA phonons detected at the side of the slab opposite to Al transducer without and with multiple reflections at the surfaces; from top to the bottom: 0, 2, 4, and 6 reflections. (b) Chirped pulses for QLA phonons for various pump powers on the Al transducer.
We now demonstrate the application of the chirped acoustic pulses to phonon spectroscopy. For this we explore the recently revealed effect of the detection gaps in sub- $\mathrm{THz}$ range when probing strain pulses by light with wavelength $\lambda_{\text {det }} \approx 400 \mathrm{~nm}$ in GaAs [30]. The dielectric function and elasto-optical constants in GaAs have resonances around this wavelength [31] and the frequency of the detection gap strongly depends on $\lambda_{\text {det }}$. Below we show how the detection gap observed as a dip in a FFT of the acoustic pulse is clearly observed as a minimum in the amplitude at a corresponding time in the chirped pulse. The results for QLA and QSTA phonons are shown in Fig. 3. Figures 3(a) and 3(c) show the temporal evolutions $\Delta R(t)$ of acoustic pulses measured for various wavelengths $\lambda_{\text {det }}$ and Figs. 3(b) and 3(d) are the FFTs obtained from the whole $\Delta R(t)$. The minima of the oscillation amplitude in $\Delta R(t)$ and the detection gaps [30] in FFT are indicated by vertical arrows. From the comparison between the temporal curves and the amplitude spectra, we find that there is a correlation between the time $t_{g}$ when the amplitude possesses minima and the corresponding frequency $f_{g}$ of the gap. It is clearly seen that $f_{g}$ and $t_{g}$ increase with the decrease of $\lambda_{\operatorname{det}}$ and the value of $f_{g}=$ $117 \mathrm{GHz}$ for QLA [Fig. 3(d)] is 1.75 times higher than $f_{g}=$ $67 \mathrm{GHz}$ for QSTA [upper curve in Fig. 3(b)] measured at the

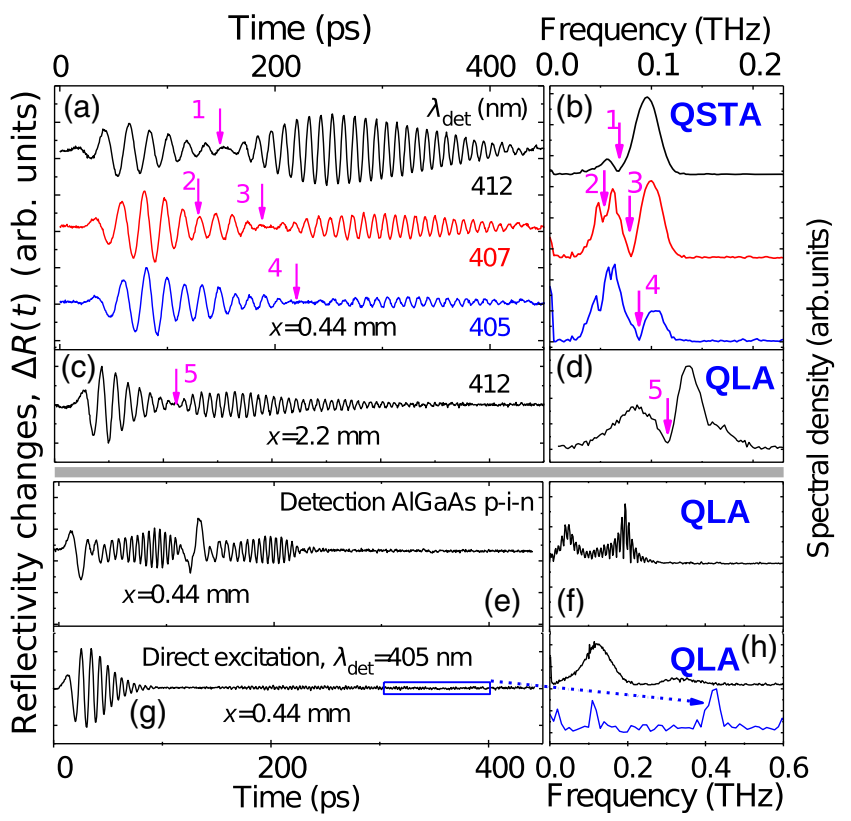

FIG. 3. (a),(b) Chirped QSTA pulses and their FFTs, respectively, for three detection wavelengths $\lambda_{\text {det }}$. (c),(d) Chirped QLA pulse and its FFT for $\lambda_{\text {det }}=412 \mathrm{~nm}$. Arrows and corresponding numbers indicate the temporal [in (a) and (c)] and spectral [in (b) and (d)] positions of the detection gaps;. (e),(f) Chirped QLA pulses and their FFTs measured with $p-i-n$ detector. (g) Chirped QLA pulse when the seed is excited by direct excitation of GaAs with blue light. (h) The spectra obtained from the pulse shown in (g) by FFT in a full 500-ps (upper curve) time window or in a 100 -ps window indicated by a blue box in $(\mathrm{g})$. 
same $\lambda_{\text {det }}=412 \mathrm{~nm}$, which is the same ratio as for velocities of QLA and QSTA sound. This confirms the model of the detection gap [30] as an example for phonon spectroscopy in the present work.

Another example of using chirped pulses in broadband $\mathrm{THz}$ phonon spectroscopy is shown in Figs. 3(e) and 3(f). Here the temporal evolution of the QLA strain pulses is measured using a $p-i-n$ diode photoconductive GaAs/ AlGaAs acoustic detector incorporating a 8-nm GaAs quantum well (QW) [25]. Two chirped acoustic pulses are observed: the first is due to the acoustic wave packet passing the QW on its way to the surface of the $p-i-n$ device and the second is due to the reflected pulse. The sharp peaks that modulate the spectrum are due to the inclusion of the reflected pulse in the FFT window. The main feature is a broad peak in the FFT at $f \approx 0.2 \mathrm{THz}$ and, related to this, the increase of the amplitude of the oscillations with time in the measured chirped pulse. This is most likely due to the frequency-dependent competing roles of piezoelectric and deformation potential mechanisms in the detection of acoustic pulses by the $p-i-n$ device fabricated on (311) [19], a detailed discussion of which is beyond the scope of the present Letter. The main conclusion of this result is that the increase of the oscillation amplitude in the measured temporal trace of the chirped acoustic pulse corresponds to the peak in the phonon spectrum around 0.2 THz. Such consistency confirms the validity of using chirped pulses for phonon spectroscopy.

Figures 3(g) and 3(h) demonstrate how THz monochromatic phonons can be extracted by time gating. Figure $3(\mathrm{~g})$ shows the QLA chirped pulse without multiple reflections $(x=0.44 \mathrm{~mm})$ detected by the photoelastic response at $\lambda_{\text {det }}=412 \mathrm{~nm}$. The upper and lower curves in Fig. 3(h) show FFTs obtained in the whole 500-ps time window and in a 100-ps time window of the tail, the gate, shown by the box in Fig. 3(g). It is seen that the gating allows us to extract quasimonochoromatic phonons with central frequency $420 \mathrm{GHz}$ and spectral width $30 \mathrm{GHz}$, which are difficult to resolve in the full-window FFT owing to the background and noise. The spectrum depends on the time window of the FFT [27]. This example shows the potential advantage of using time gating in phonon spectroscopy under conditions where the noise makes it impossible to extract the spectral features in a certain frequency domain. The frequency and the width of the gated phonon spectrum can be selected by choosing the temporal position of the gate and its width. In the Supplemental Material [27] we show a simulated example where we compare the effects of a notch filter on the spectrum of nonchirped and chirped acoustic pulses. We compare the results with the absence and presence of jitter noise which is always present in pump-probe experiments and defines the ultimate temporal resolution of the system.

In summary, we have demonstrated the following: (i) a method to generate and control up-chirped acoustic pulses for sub-THz phonons with three polarizations on propagation distances $\sim 1 \mathrm{~mm}$; (ii) the compliance of the envelope function in the measured temporal trace with the phonon spectrum; (iii) an algorithm, on the basis of analytical solution of Eq. (1), for extracting the phonon spectrum directly from the oscillation amplitude, which essentially simplifies the analysis of the results compared to the method used in Ref. [17]; (iv) extraction of quasimonochromatic $\mathrm{THz}$ acoustic wave packets with tunable frequency and spectral width by time gating of the chirped signal; (v) the chirping for TA phonons, which is a significant step forward in phonon physics taking into account the big role of shear perturbations in electronphonon and magnon-phonon interactions, light scattering, piezoelectric effect, and nanoscopy of noncrystalline nanoobjects and liquids.

Our results point the way to the development of techniques utilizing chirped acoustic pulses to measure the sub- $\mathrm{THz}$ and $\mathrm{THz}$ phonon spectra of single micro- and nano-objects, where, currently, the signal-to-noise ratio strongly limits the spectral resolution and the speed of obtaining the information. Using a chirped pulse, the measurement time window can be selected to exclude adjacent frequency components and, importantly, their phase and jitter noise contributions. The improvements, therefore, come from the reduction in integration or averaging time required at each sampling point. Sub$\mathrm{THz}$ spectroscopy with TA phonons, whose spectrum is more sensitive to defects, could provide new information about biological objects like single cells [32], and become a new instrument for nanoscopy with spectral resolution. Heterodyne mixing of a single-frequency microwave probe with chirped acoustic pulses could be used for analyzing the spectra using $\mathrm{GHz}$ oscilloscopes and spectrum analyzers [33]. Using piezoelectric transformation of $\mathrm{THz}$ acoustic waves to $\mathrm{THz}$ electromagnetic waves, e.g., Ref. [34], chirped acoustic pulses may be transformed into chirped microwave signals, becoming also an instrument for $\mathrm{THz}$ microwave spectroscopy.

This work was supported by the Engineering and Physical Sciences Research Council [Grant Number EP/ M016161/1]; and U.S. Army Research Laboratory [Grant Number W911NF-14-1-0586]. We acknowledge V. Gusev for useful discussions.

* Corresponding author. andrey.akimov@nottingham.ac.uk

[1] A. Crut, P. Maioli, N. Del Fatti, and F. Vallée, Phys. Rep. 549, 1 (2015).

[2] J. A. Rogers, A. A. Maznev, M. J. Banet, and K. A. Nelson, Annu. Rev. Mater. Sci. 30, 117 (2000).

[3] P.-A. Mante, Y.-R. Huang, S.-C. Yang, T.-M. Liu, A. A. Maznev, J.-K. Sheu, and C.-K. Sun, Ultrasonics 56, 52 (2015). 
[4] C. Thomsen, H. T. Grahn, H. J. Maris, and J. Tauc, Phys. Rev. B 34, 4129 (1986).

[5] O. Matsuda, M. C. Larciprete, R. L. Voti, and O. B. Wright, Ultrasonics 56, 3 (2015).

[6] W. Chen, Y. Lu, H. J. Maris, and G. Xiao, Phys. Rev. B 50, 14506 (1994).

[7] M. F. Pascual-Winter, A. Fainstein, B. Jusserand, B. Perrin, and A. Lemaître, Phys. Rev. B 85, 235443 (2012).

[8] O. Matsuda, O. B. Wright, D. H. Hurley, V. Gusev, and K. Shimizu, Phys. Rev. B 77, 224110 (2008).

[9] P. M. Walker, A. J. Kent, M. Henini, B. A. Glavin, V. A. Kochelap, and T. L. Linnik, Phys. Rev. B 79, 245313 (2009).

[10] T. Pezeril, P. Ruello, S. Gougeon, N. Chigarev, D. Mounier, J.-M. Breteau, P. Picart, and V. Gusev, Phys. Rev. B 75, 174307 (2007).

[11] M. Lejman, G. Vaudel, I. C. Infante, P. Gemeiner, V. E. Gusev, B. Dkhil, and P. Ruello, Nat. Commun. 5, 4301 (2014).

[12] Y.-C. Wen, T.-S. Ko, T.-C. Lu, H.-C. Kuo, J.-I. Chyi, and C.-K. Sun, Phys. Rev. B 80, 195201 (2009).

[13] T. Pezeril, N. Chigarev, P. Ruello, S. Gougeon, D. Mounier, J.-M. Breteau, P. Picart, and V. Gusev, Phys. Rev. B 73, 132301 (2006).

[14] A. Bartels, R. Cerna, C. Kistner, A. Thoma, F. Hudert, C. Janke, and T. Dekorsy, Rev. Sci. Instrum. 78, 035107 (2007).

[15] G. B. Park and R. W. Field, J. Chem. Phys. 144, 200901 (2016).

[16] R. Kaltenbaek, J. Lavoie, D. N. Biggerstaff, and K. J. Resch, Nat. Phys. 4, 864 (2008).

[17] H.-Y. Hao and H. J. Maris, Phys. Rev. B 63, 224301 (2001).

[18] Electron-Phonon Interactions in Low-Dimensional Structures, edited by L. J. Challis (Oxford University Press, Oxford, 2003), Vol. 10.

[19] D. Srikanthreddy, B. A. Glavin, C. L. Poyser, M. Henini, D. Lehmann, C. Jasiukiewicz, A. V. Akimov, and A. J. Kent, Phys. Rev. Applied 7, 024014 (2017).

[20] C. Kittel, Introduction to Solid State Physics (Wiley, New York, 2005)
[21] M. Bombeck, J. V. Jäger, A. V. Scherbakov, T. Linnik, D. R. Yakovlev, X. Liu, J. K. Furdyna, A. V. Akimov, and M. Bayer, Phys. Rev. B 87, 060302 (2013).

[22] V. L. Korenev, M. Salewski, I. A. Akimov, V. F. Sapega, L. Langer, I. V. Kalitukha, J. Debus, R. I. Dzhioev, D. R. Yakovlev, D. Müller et al., Nat. Phys. 12, 85 (2016).

[23] A. V. Scherbakov, M. Bombeck, J. V. Jäger, A. S. Salasyuk, T. L. Linnik, V. E. Gusev, D. R. Yakovlev, A. V. Akimov, and M. Bayer, Opt. Express 21, 16473 (2013).

[24] A. P. Sarvazyan, O. V. Rudenko, S. D. Swanson, J. B. Fowlkes, and S. Y. Emelianov, Ultrasound Med. Biol. 24, 1419 (1998).

[25] D. Moss, A. V. Akimov, R. P. Campion, M. Henini, C. T. Foxon, L. Eaves, A. J. Kent, and B. A. Glavin, Phys. Rev. B 83, 245303 (2011).

[26] W. Chen, H. J. Maris, Z. R. Wasilewski, and S.-I. Tamura, Philos. Mag. B 70, 687 (1994).

[27] See Supplemental Material at http://link.aps.org/ supplemental/10.1103/PhysRevLett.119.255502 for a description of the experimental scheme and theoretical analysis of the data.

[28] H.-Y. Hao and H. J. Maris, Phys. Rev. B 64, 064302 (2001).

[29] E. Péronne, N. Chuecos, L. Thevenard, and B. Perrin, Phys. Rev. B 95, 064306 (2017).

[30] C. He, O. Ristow, M. Grossmann, D. Brick, Y. Guo, M. Schubert, M. Hettich, V. Gusev, and T. Dekorsy, Phys. Rev. B 95, 184302 (2017).

[31] P. Etchegoin, J. Kircher, M. Cardona, C. Grein, and E. Bustarret, Phys. Rev. B 46, 15139 (1992).

[32] C. Rossignol, N. Chigarev, M. Ducousso, B. Audoin, G. Forget, F. Guillemot, and M. C. Durrieu, Appl. Phys. Lett. 93, 123901 (2008).

[33] S. L. Heywood, B. A. Glavin, R. P. Beardsley, A. V. Akimov, M. W. Carr, J. Norman, P. C. Norton, B. Prime, N. Priestley, and A. J. Kent, Sci. Rep. 6, 30396 (2016).

[34] M. R. Armstrong, E. J. Reed, K.-Y. Kim, J. H. Glownia, W. M. Howard, E. L. Piner, and J. C. Roberts, Nat. Phys. 5, 285 (2009). 